\title{
Review: apolipoprotein $E \in 4$ allele is a risk factor for Alzheimer's disease in different ethnic groups
}

\author{
Farrer LA, Cupples LA, Haines JL, et al, for the APOE and Alzheimer Disease Meta Analysis Consortium. Effects of age, sex, and \\ ethnicity on the association between apolipoprotein E genotype and Alzheimer disease.JAMA 1997 Oct 22;278:1349-56.
}

\section{Question}

What is the effect of age, sex, and ethnicity on the association between apolipoprotein E (APOE) genotype and Alzheimer's disease $(\mathrm{AD})$ ?

\section{Data sources}

A Medline search was done to assist in identifying research groups known to be investigating the association between APOE $\epsilon 4$ allele and $\mathrm{AD}$. The research groups were invited to provide data sets on their patients.

\section{Study selection}

The data sets included patients who had definite or probable AD (5930 patients). Patients with mutations in the amyloid precursor protein gene and the presenilin genes or coexisting neuropathological problems were excluded. Control group participants had no neurodegenerative or neuropsychiatric illnesses (8607 patients)

\section{Data extraction}

The data sets from the centres were reviewed for sampling strategies (cohort, case control, and cross sectional designs); recruitment settings (community, clinic or hospital, and autopsy or brain bank); and the ethnic or racial designations of the case and control subjects. In addition, the diagnostic status, sex, age at last examination or death, age at disease onset, family history, and APOE genotype were determined for each subject. The odds ratios (ORs) for $\mathrm{AD}$ adjusted for age and study were determined for APOE genotypes with the genotype $\epsilon 3 / € 3$ as the referent group.

\begin{abstract}
Main results
Among white patients from clinic or autopsy studies, the risk of $\mathrm{AD}$ was greater in patients with genotypes $\epsilon 2 / \epsilon 4(\mathrm{OR} 2.6,95 \%$ CI 1.6 to 4.0 ); $\epsilon 3 / \epsilon 4$ (OR 3.2, CI 2.8 to 3.8); and $\epsilon 4 / \epsilon 4$ (OR 14.9 , CI 10.8 to 20.6 ) and the risk was decreased in patients with genotype $\epsilon 2 / \epsilon 3$ (OR 0.6 , CI 0.5 to 0.8 ). Similarly, in population based studies of white patients, the $\epsilon 3 / \epsilon 4$ and $\epsilon 4 / \epsilon 4$ genotypes were associated with $\mathrm{AD}$ (OR 2.7, CI 2.2 to 3.2 and OR 12.5 CI 8.8 to 17.7 , respectively) and the $\epsilon 2 / \epsilon 3$ genotype was protective (OR 0.6 , CI 0.5 to 0.9). The association between the $\in 4 / \in 4$ genotype and $\mathrm{AD}$ was strongest among Japanese patients (OR 33.1, CI 13.6 to 80.5) and weakest among African-American (OR 5.7, CI 2.3 to 14.1) and Hispanic patients (OR 2.2, CI 0.7 to 6.7). Substantial heterogeneity existed in studies of AfricanAmerican patients. In white patients with the $\epsilon 3 / \epsilon 4$ or $\epsilon 4 / \epsilon 4$ genotypes, the risk of $\mathrm{AD}$ increased between 40 and 60 years of age. Age affected Japanese patients with the $\epsilon 3 / € 4$ similarly but did not affect risk in Hispanic patients. The risk of $\mathrm{AD}$ in patients with the $\epsilon 3 / \in 4$ and $\in 2 / \epsilon 4$ genotypes, compared with those with the $\in 3 / € 3$ genotype, was greater in women than in men.
\end{abstract}

\section{Conclusions}

The apolipoprotein $\mathrm{E} \in 4$ allele is strongly associated with Alzheimer's disease in white, African-American, Hispanic, and Japanese people. The risk is evident between 40 and 90 years of age.

Sources of funding: Alzheimer Association; Athena Diagnostics, Inc; National Institutes of Health.

For correspondence: $\operatorname{Dr} L$ A Farrer, Department of Neurology, Boston University School of Medicine, 80 E Concord Street, Boston, MA O2118, USA. Fax +1 6176384275.

\section{Commentary}

A positive association between the $\epsilon 4$ allele of the APOE gene and AD was first reported in 1993. It has become the most statistically robust and biologically important example of a genetic risk factor for a common psychiatric disorder. As such, the main conclusion of the metaanalysis by Farrer $e t$ al, that the APOE $\in 4$ allele is a determinant of risk of $\mathrm{AD}$, is hardly unexpected. The real value of this review is that it clarifies the magnitude of the risk in population based and in clinic based samples, and it highlights some of the complexities in the association. For example, it confirms suspicions that possession of an $\epsilon 2$ is protective, but shows that this effect is more than cancelled out if $\epsilon 2$ is inherited with an $\epsilon 4$ allele. The meta-analysis also makes clear that the size, although not the existence, of risk conferred by the $\epsilon 4$ allele differs considerably not only between groups but also within the African-American population (which is usually considered as a single entity in genetic research). Establishing these details about the influence of APOE on AD provides clearer pointers as to populations and experimental strategies which may be informative when looking for additional genetic and environmental contributors to the disease.

The APOE association is of undoubted importance to the understanding of $\mathrm{AD}$, ranking alongside the seminal discoveries of the cholinergic deficits and the genes causing the dominant familial form of the disease. This meta-analysis should not, however, be seen as bearing directly upon the possible use of APOE testing in the differential diagnosis of $\mathrm{AD}$, let alone the prediction of its occurrence or its response to treatment. ${ }^{1-3}$ These crucial issues await clarification about the sensitivity, specificity, and predictive value of APOE testing in each of these clinically relevant situations. This information requires more extensive research in several areas, including the association of the APOE genotype with other disorders, and prospective studies of younger, cognitively normal cohorts.

$$
\begin{array}{r}
\text { Paul Harrison, MA, DM, MRCPysch } \\
\text { Warneford Hospital } \\
\text { Oxford, UK }
\end{array}
$$

1 National Institute on Aging/Alzheimer's Association Working Group. Apolipoprotein E genotyping in Alzheimer's disease. Lancet 1996;347:1091-5.

2 Saunders AM, Hulette O, Welsh-Bohmer KA, et al. Specificity, sensitivity, and predictive value of apolipoprotein-E genotyping for sporadic Alzheimer's disease. Lancet 1996;348:90-3.

3 Richard F, Helbecque N, Neuman $\mathrm{F}$ et al. APOE F, Helbecque N, Neuman E, et al. APOE genotyping and response to drug 349.539. 